\title{
Evaluation of the Occupational Doses of Interventional Radiologists
}

\author{
Gerritjan Kuipers · Xandra L. Velders • \\ Robbert J. de Winter · Jim A. Reekers • \\ Jan J. Piek
}

Received: 10 April 2007/Accepted: 15 January 2008/Published online: 12 February 2008

(C) The Author(s) 2008

\begin{abstract}
The aim of the present study was to determine whether there is a linear relation between the doses measured above and those measured under the lead apron of the radiologists performing interventional procedures. To monitor radiation exposure the International Commission of Radiological Protection (ICRP) recommends the use of a single dosimeter under the protective apron. To determine the exposure more accurately an additional dosimeter is recommended above the protective apron. The exposure of eight radiologists was monitored with two personal dosimeters during 3 consecutive years. To measure the doses uniformly the two dosimeters were worn in a special holder attached to the lead apron. The two personal dosimeters were replaced every 4 weeks on the same day. The doses above and under the protective aprons of seven radiologists did not differ significantly. A significant lower dose above and under the protective apron was measured for one of the radiologists. During a 4-week period the average dose measured above the lead apron was $3.44 \mathrm{mSv}$ (median, $3.05 \mathrm{mSv}$ ), while that under the $0.25-\mathrm{mm}$ lead apron was $0.12 \mathrm{mSv}$ (median, $0.1 \mathrm{mSv}$ ). The coefficients of the regression line result in the equation $Y=0.036 X-0.004$, with $Y$ as the dose under the lead apron and $X$ as the dose
\end{abstract}

G. Kuipers $(\bowtie) \cdot$ X. L. Velders

Radiation Protection Group, Academic Medical Center, Meibergdreef 9, Amsterdam 1105 AZ, The Netherlands e-mail: g.kuipers@amc.uva.nl

R. J. de Winter · J. J. Piek

Department of Cardiology, Academic Medical Center, Meibergdreef 9, Amsterdam 1105 AZ, The Netherlands

\section{J. A. Reekers}

Department of Radiology, Academic Medical Center, Meibergdreef 9, Amsterdam 1105 AZ, The Netherlands above the lead apron. The statistical analysis of the data established a linear relation between the doses above and those under the lead apron $\left(R^{2}=0.59\right)$. Before the special holder was introduced it was not possible to derive a relation between the doses above and those under the lead apron, as the doses were measured at varying places above and under the lead apron. There is no evidence that the effective dose can be estimated more accurately when an additional dosimeter is used. The present study revealed a threshold before doses under the lead apron were measured. Due to the threshold it can be concluded that the doses under the lead apron will not be underestimated easily when doses above the lead apron are used to calculate them. This is not the case when the doses above the lead apron are calculated for the doses under the lead apron.

Keywords Dosimetry - Fluoroscopy .

Radiation protection $\cdot$ Effective dose $\cdot$ Lead apron

\section{Introduction}

Like many other hospitals the Academic Medical Centre (AMC) in Amsterdam uses personal dosimeters to monitor the occupational doses from $\mathrm{x}$-ray procedures to clinical staff. The occupational doses of the majority of the clinical staff do not exceed $1 \mathrm{mSv}$ in a single year. However, for clinical staff involved in fluoroscopically guided interventional procedures, significant doses are measured. As a consequence of the radiation exposure, the risk of fatal cancer for the radiologists performing interventional procedures increases. This stochastic risk can increase to a value of about $0.1 \%$ for radiologists performing these procedures during many years [1]. In recent years the number of fluoroscopically guided interventional procedures at the 
AMC has increased, as has the number of radiologists performing the procedures. Moreover, the discipline to wear personal dosimeters has improved. As a result, the number of radiologists with doses exceeding $20 \mathrm{mSv}$ in a single year has increased. Whenever the dose exceeds $20 \mathrm{mSv}$ in a single year a report has to be sent to the Dutch labor inspection, even when the doses are measured above the lead apron. In the report evidence has to be given that the limit for stochastic effects is not exceeded. In order to prove that the doses of $20 \mathrm{mSv}$ measured above lead aprons do not exceed the stochastic dose limit, it was decided to provide additional personal dosimeters to the radiologists performing interventional procedures at the AMC. These additional personal dosimeters were meant to monitor the occupational doses under the lead apron.

When in 2004 it was decided to provide the radiologists with an additional dosimeter, the AMC had already substantial data of doses monitored on both sides of the lead apron. These data were collected at the Department of Cardiology, where additional dosimeters were introduced in 1998. Until 2004 the data collected at the Department of Cardiology established no relation between the doses measured above and those measured under the lead apron. This was thought to be caused by the fact that the two dosimeters were often transposed, that the dosimeters were not always returned at the same time, and that unmistakable instruction regarding the position of the two personal dosimeters was missing. To rule out the influence of these factors on the dose measured, it was decided to develop a special holder for the two dosimeters at the time the radiologists were provided with additional dosimeters. Moreover, it was decided to replace all personal dosimeters in the study on the same day by one person, to preclude the influence of differences in measuring periods and problems with transposed dosimeters.

For this department the doses measured under the lead apron were considered to be a substitute for the effective dose as recommended by the ICRP [2], while the doses measured above the lead apron were used to estimate the effective dose more accurately.

The aim of the present study was to determine whether a relation exists between the doses measured above and those measured under the lead aprons of radiologists performing interventional procedures and whether two personal dosimeters provide additional information compared to a single dosimeter.

\section{Materials and Methods}

From 2004 onward the occupational doses of eight radiologists involved in interventional fluoroscopically guided procedures were monitored by means of two personal dosimeters. The personal dosimeters were used to measure the doses above and under the lead aprons of the radiologists. To receive consistent measurements the two dosimeters were worn in a specially designed holder that was fixed to the lead apron (Figs. 1 and 2). The holder with the two personal dosimeters was worn breast-high. The two dosimeters were replaced every 4 weeks on the same day, while the holder remained fixed to the lead apron during the whole study.

The doses were measured during 39 periods of 4 weeks in 3 consecutive years.

At the start of the study six of the radiologists were provided with two personal dosimeters, while throughout the study another two radiologists were provided with two dosimeters. Three radiologists left the AMC before the end of the study.

The personal dosimeters were provided by the Nuclear Research and Consultant Group (NRG, Arnhem, the Netherlands). The personal dosimeters were read by the NRG and reported to the AMC. The dosimeters contain LiF:Ti, Mg (TLD100) detectors behind $2 \mathrm{~mm}$ of aluminum for the measurements at a depth of $10 \mathrm{~mm}$. In the dose reports the doses were presented by means of the personal dose equivalent at a depth of $10 \mathrm{~mm}$, the $\mathrm{H}_{\mathrm{p}}(10)$. The doses were reported in multiples of $0.01 \mathrm{mSv}$. Doses below $0.005 \mathrm{mSv}$ were reported as $<0.01 \mathrm{mSv}$ [3].

At the Department of Radiology the interventional procedures were performed with two Philips Integris Allura machines (Philips Medical Systems, Best, the Netherlands) with the same potential range of $50-133 \mathrm{kVp}$. During all interventional procedures the radiologists used wraparound lead aprons (skirts and vests; Scanflex Medical AB, Täby, Sweden) with an overall lead equivalence of $0.25 \mathrm{~mm}$ at $100 \mathrm{kV}$. The wrap-around apron has an overlap due to which the actual thickness of the protective apron in the front is $0.5 \mathrm{~mm}$ lead equivalent. The doses were

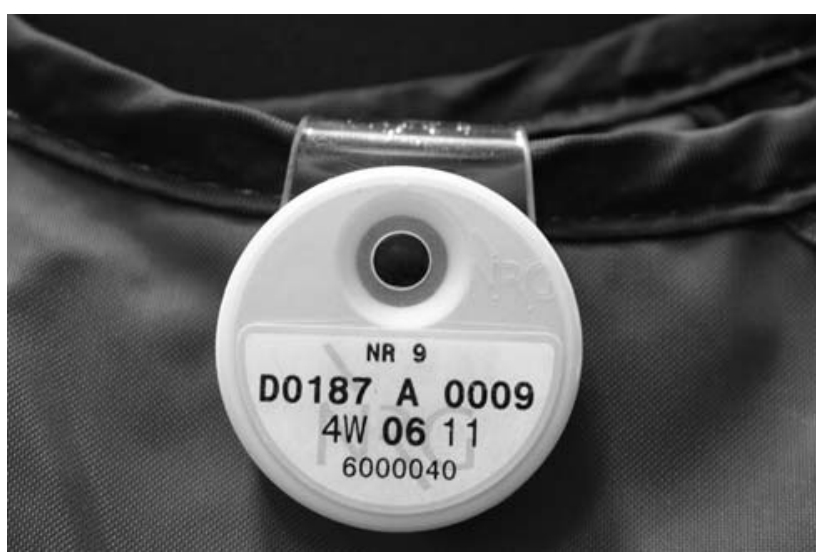

Fig. 1 The specially designed holder fixed to the lead apron breasthigh 


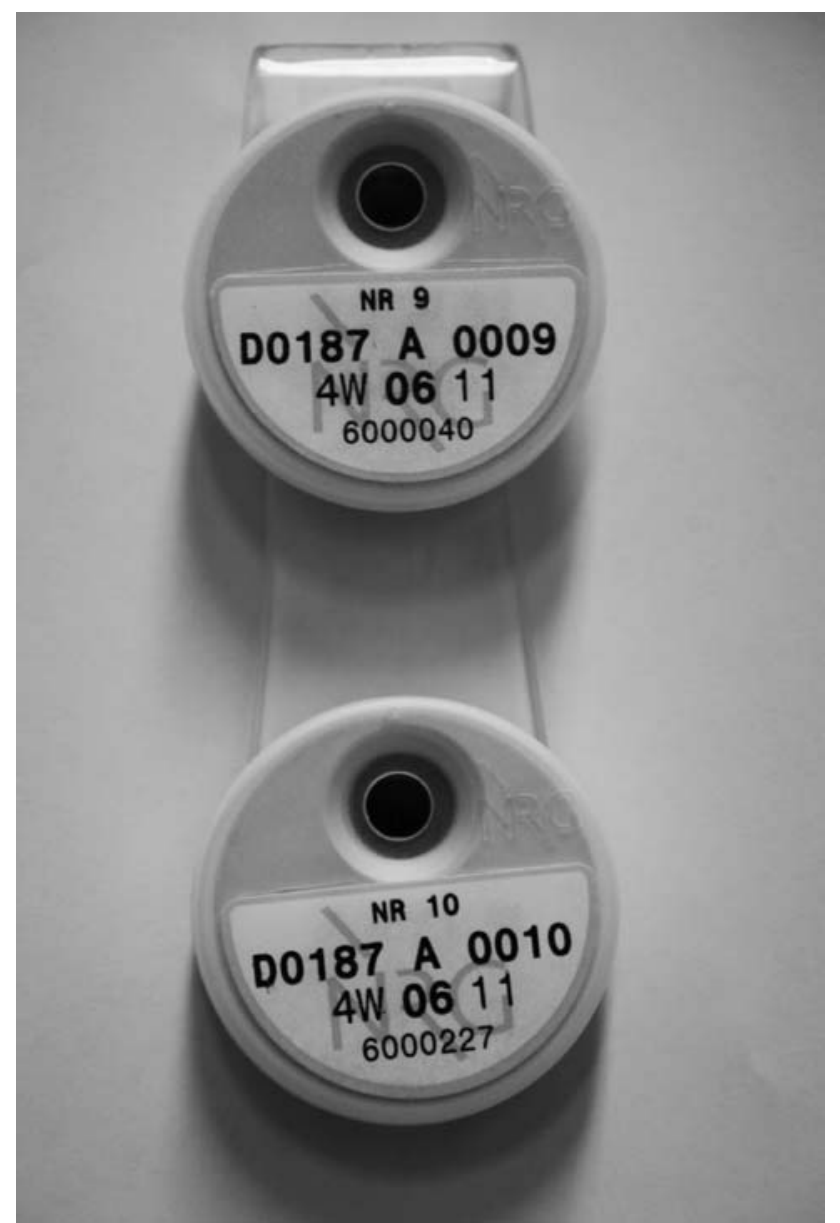

Fig. 2 The specially designed holder with the two personal dosimeters

monitored above and under a layer of $0.25 \mathrm{~mm}$ lead equivalence.

The doses measured above and under the lead apron were statistically analyzed using SPSS software (release 11.5.1 for Windows). Under the lead apron doses
$<0.01 \mathrm{mSv}$ were treated as $0 \mathrm{mSv}$ for the statistical analysis. Above the lead apron doses $<0.01 \mathrm{mSv}$ were entered as missing values. For the statistical analysis the doses under the lead apron were entered as dependent variables, while the doses above the lead apron and the eight radiologists were independent variables. Statistical significant differences were set at a level of 5\% $(p=0.05)$.

\section{Results}

During the study 392 dosimeters were provided to eight radiologists. All dosimeters were returned to the NRG. The doses were read and reported in 134 -weekly dose reports. In total 196 doses were recorded above the lead apron, ranging from $<0.01$ to $16.78 \mathrm{mSv}$. Under the lead apron the doses ranged from $<0.01$ to $0.83 \mathrm{mSv}$. Five readings were excluded from the study, as the doses measured above and under the lead apron were reported to be $<0.01 \mathrm{mSv}$. In 27 cases the doses measured under the lead apron were $<0.01 \mathrm{mSv}$. These doses were treated as $0 \mathrm{mSv}$ in the statistical analysis.

In Tables 1 and 2 and in Figs. 3 and 4, the mean dose, median, and quartiles above and under the lead apron are presented for the eight radiologists. A KolmogorovSmirnov test showed that the doses of the eight radiologists were normally distributed $(p>0.05)$. The doses measured above and under the lead apron differed significantly among the eight radiologists (one-way ANOVA, $p<0.05$ ). The Levenes test established that the variance of the doses measured above and under the lead apron of seven radiologists was equal $(p>0.05)$. The doses of these seven radiologists did not differ significantly (one-way ANOVA, $p>0.05$ ). The average dose of the seven radiologists was $3.85 \mathrm{mSv}$ in 4 weeks above the lead apron and $0.13 \mathrm{mSv}$ in 4 weeks under the lead apron.

Table 1 The number of measurements, mean dose, standard deviation, and percentiles (25th, 50th [median], and 75th) of the doses measured above the lead aprons of radiologists 1 to 8

\begin{tabular}{lrllllllr}
\hline Radiologist no. & $N$ & Mean $(\mathrm{mSv})$ & SD & 25th $(\mathrm{mSv})$ & 50th $(\mathrm{mSv})$ & 75th $(\mathrm{mSv})$ & Minimum (mSv) & Maximum $(\mathrm{mSv})$ \\
\hline 1 & 4 & 3.41 & 1.22 & 2.16 & 3.58 & 4.48 & 1.88 & 4.58 \\
2 & 34 & 3.67 & 3.21 & 1.32 & 3.15 & 5.26 & 0.03 & 16.78 \\
3 & 9 & 2.33 & 1.24 & 1.38 & 2.17 & 3.59 & 0.28 & 4.00 \\
4 & 9 & 3.65 & 2.61 & 1.76 & 3.56 & 4.72 & 0.35 & 9.24 \\
$5^{\text {a }}$ & 38 & 1.79 & 1.09 & 0.88 & 1.70 & 2.29 & 0.14 & 5.40 \\
$6^{\text {b }}$ & 39 & 3.68 & 2.37 & 2.25 & 3.26 & 4.18 & 0.13 & 0.66 \\
$7^{\text {b }}$ & 21 & 3.66 & 1.71 & 2.49 & 3.08 & 4.57 & 1.52 \\
$8^{\text {b }}$ & 37 & 4.75 & 2.31 & 3.10 & 4.87 & 6.13 & 1.05 & 13.23 \\
Total & 191 & 3.44 & 2.41 & 1.74 & 3.05 & 4.61 & 0.03 & 16.78 \\
\hline
\end{tabular}

Note. Doses followed by a superscript a differ significantly from doses followed by a superscript $\mathrm{b}(p<0.05)$. 
Table 2 The number of measurements, mean dose, standard deviation, and percentiles (25th, 50th [median], and 75th) of the doses measured under the lead aprons of radiologists 1 to 8

\begin{tabular}{lrlllllll}
\hline Radiologist no. & $N$ & Mean $(\mathrm{mSv})$ & SD & 25th $(\mathrm{mSv})$ & 50th $(\mathrm{mSv})$ & 75th $(\mathrm{mSv})$ & Minimum $(\mathrm{mSv})$ & Maximum $(\mathrm{mSv})$ \\
\hline 1 & 4 & 0.18 & 0.09 & 0.10 & 0.17 & 0.27 & 0.09 & 0.29 \\
2 & 34 & 0.13 & 0.15 & 0.03 & 0.11 & 0.17 & 0.00 & 0.83 \\
3 & 9 & 0.06 & 0.06 & 0.01 & 0.07 & 0.11 & 0.00 & 0.15 \\
4 & 9 & 0.14 & 0.09 & 0.06 & 0.15 & 0.21 & 0.00 & 0.24 \\
$5^{\text {a }}$ & 38 & 0.07 & 0.09 & 0.00 & 0.06 & 0.09 & 0.00 & 0.49 \\
6 & 39 & 0.12 & 0.08 & 0.06 & 0.11 & 0.19 & 0.00 & 0.37 \\
$7^{\text {a }}$ & 21 & 0.20 & 0.12 & 0.12 & 0.18 & 0.27 & 0.02 & 0.50 \\
8 & 37 & 0.13 & 0.12 & 0.05 & 0.12 & 0.18 & 0.00 & 0.66 \\
Total & 191 & 0.12 & 0.11 & 0.05 & 0.1 & 0.17 & 0.00 & 0.83 \\
\hline
\end{tabular}

a These doses differ significantly $(p<0.05)$.

The average dose of radiologist 5 was $1.79 \mathrm{mSv}$ in 4 weeks above the lead apron and $0.07 \mathrm{mSv}$ under the lead apron. The dose above the lead apron of radiologist 5 differed significantly from the doses of radiologists 6,7 , and 8 (Tamhane post hoc analysis, $p<0.05$ ). Under the lead apron the dose of radiologist 5 differed significantly from the dose of radiologist 7 (Tamhane post hoc analysis, $p<0.05)$.

In Fig. 5 the doses measured under the lead apron are plotted against the doses above the lead apron. The statistical analysis of the data established a linear relation

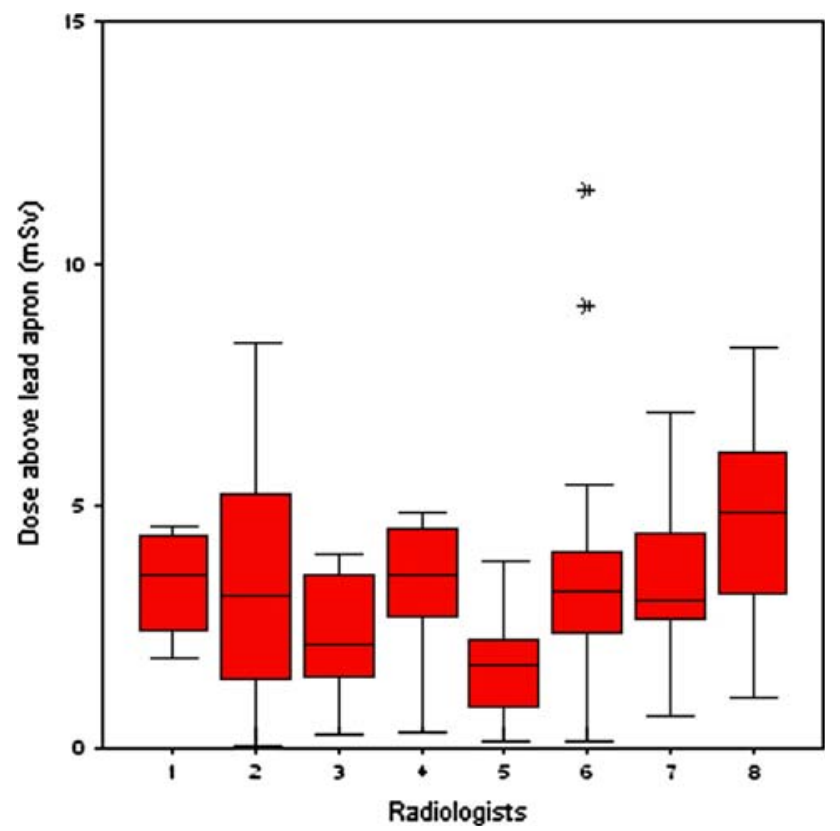

Fig. 3 Boxplot of the doses (mSv) measured above the lead apron. The black line in the red box marks the median, the box demarks the 25th and 75th percentiles, and the whiskers mark the minimum and maximum observed values that are not statistical outliers. Extreme values are marked with an asterisk. The extreme values are more than three box lengths from the upper edge of the box. The box length is the interquartile range between the doses above and those under the lead apron (ANOVA, $p<0.05, R^{2}=0.59$ ). The coefficients of the regression line representing the relation between the doses measured above and those measured under the leaded apron were derived, resulting in the equation $Y=0.036 X$ -0.004 , with $Y$ as the dependent variable (dose under the lead apron) and $X$ as the predictor value (dose above the lead apron). The $95 \%$ confidence interval for the coefficients ranged from 0.034 to 0.038 . The standard error of the models tested was 0.07 . The standard error of the model was lower than the standard deviation of the average dose

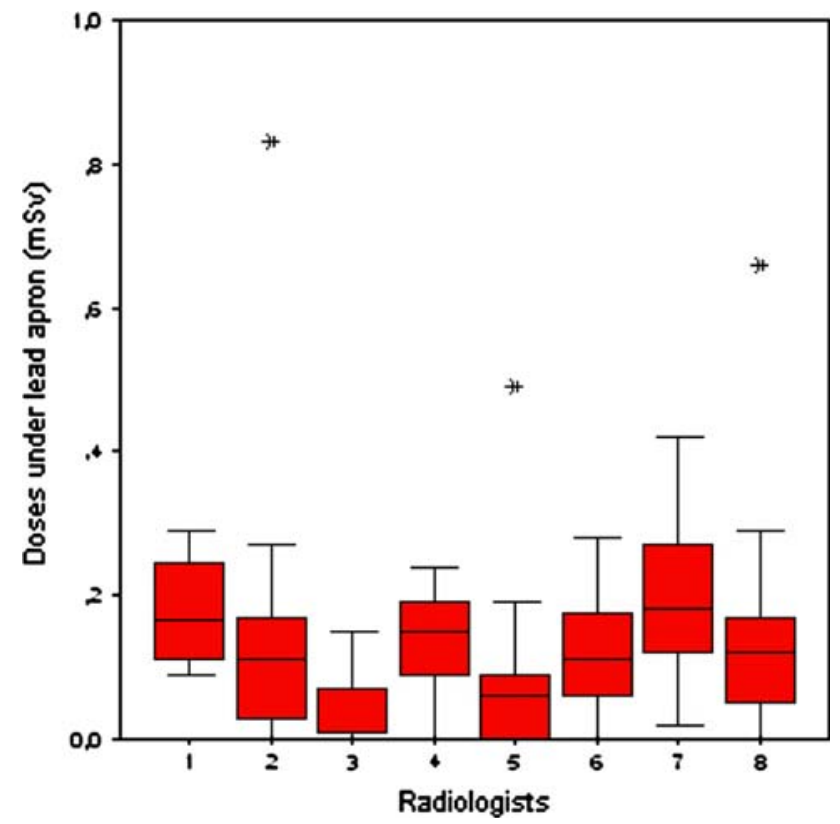

Fig. 4 Boxplot of the doses (mSv) measured under the lead apron. The black line in the red box marks the median, the box demarks the 25th and 75th percentiles, and the whiskers mark the minimum and maximum observed values that are not statistical outliers. Extreme values are marked by an asterisk. The extreme values are more than three box lengths from the upper edge of the box. The box length is the interquartile range 


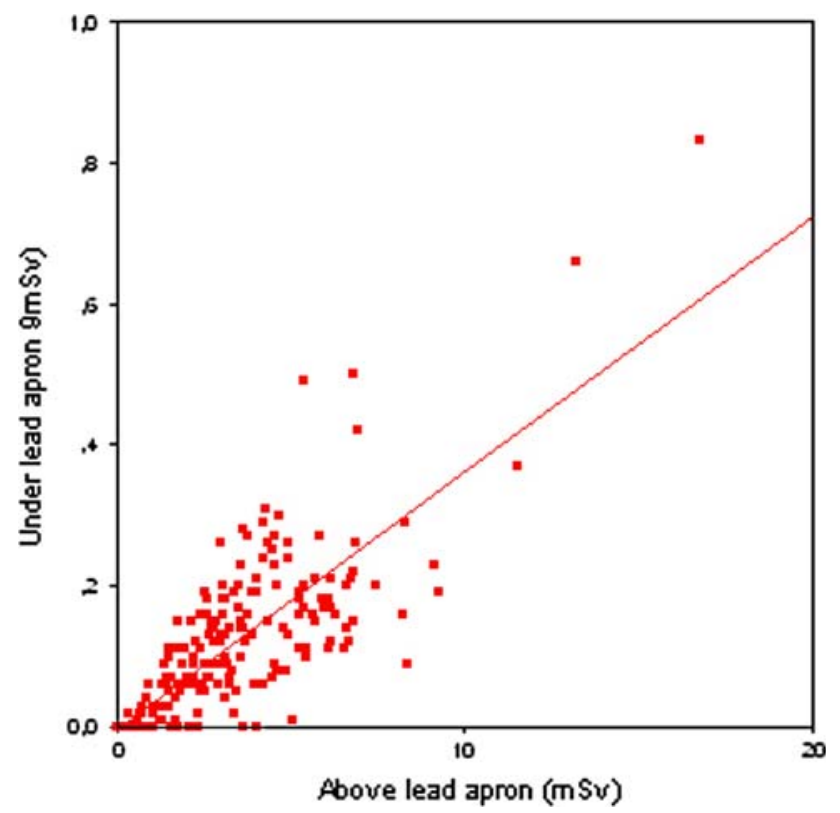

Fig. 5 Plot of doses $(\mathrm{mSv})$ measured above the lead apron ( $X$-axis) and under the lead apron ( $Y$-axis). The line represents the equation for the regression

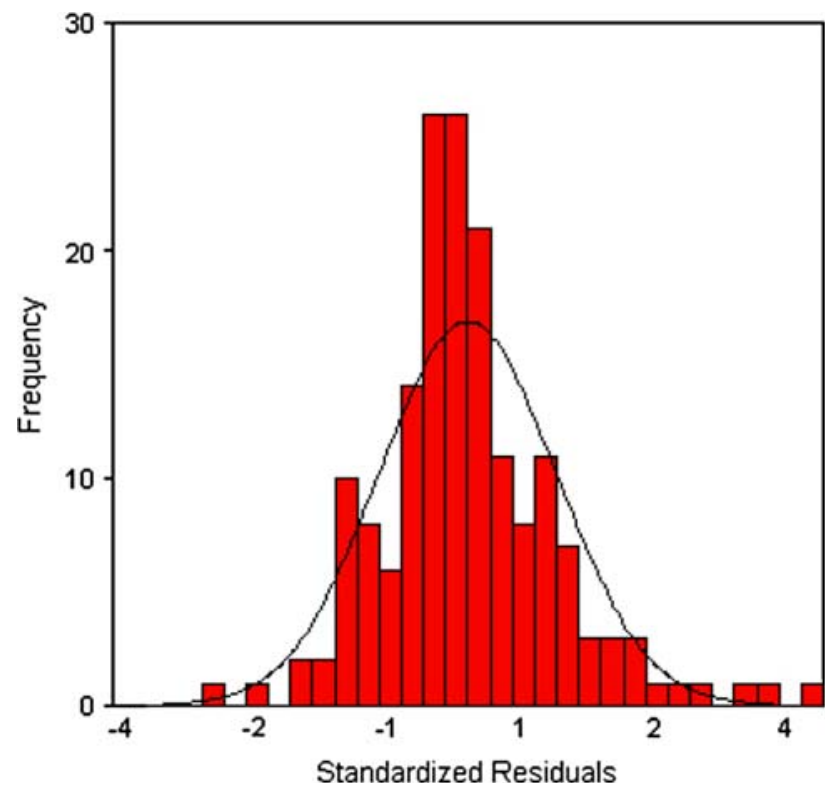

Fig. 6 Distribution of the residuals $\quad$ (mean $=0$, standard deviation $=1$ )

under the lead apron (0.11). The residuals were normally distributed $(p>0.05)$ (Fig. 6).

The linear relation was not influenced by the doses measured for radiologist 5 . The equation remained the same. With the equation and the lowest detectable dose under the lead apron $(<0.005 \mathrm{mSv})$, the corresponding dose above the lead apron was calculated: $<0.25 \mathrm{mSv}$. Above this threshold the relation between doses above and those under the lead apron was determined another time. The statistical analysis once again established a linear relation (ANOVA, $p<0.05$ ). The coefficients of the regression line remained the same. Only the strength of the model was lower $\left(R^{2}=0.57\right)$.

\section{Discussion}

The dose measurements above and under the lead apron established that the occupational exposure of the radiologists performing interventional procedures in the AMC is comparable. There was only one exception: radiologist 5 . As radiologist 5 performed fewer procedures than his colleagues, his exposure was expected to be lower. It was, however, unknown what reduction in doses was to be expected, as data on the number and the length of procedures were not collected for the study. Despite this, it is likely that the lower exposure of radiologist 5 was caused by a lower workload, as the doses above and under the lead apron decreased proportionally compared to the other radiologists': $46 \%$ and $50 \%$.

Results of doses measured above and under the lead apron of physicians performing interventional procedures have been published by several authors. Moreover, measurements for cardiologists performing interventional procedures were carried out. Renaud et al. [4] reported measurements above and under the lead aprons of in-room personnel during cardiac catheterization. Vano et al. [5] published measurements of cardiologists during a 15-year follow-up. However, differences in the way radiologists and cardiologists perform interventional procedures make it difficult to compare the doses of cardiologists and radiologists. A limited number of clinical studies on doses above and under the lead apron have been carried out among radiologists. Williams et al. [6] reported average monthly doses above the lead aprons of radiologists that ranged from 1.08 to $6.55 \mathrm{mSv}$. The highest average dose in this study was found for a radiologist who performed all interventional procedures in patients with liver disease. The average dose of the other radiologists in the study was $1.56 \mathrm{mSv}$. The findings of Williams et al. are comparable to the average dose above the lead apron in the present study, where the doses varied between 1.09 and $3.21 \mathrm{mSv}$.

Niklason et al. [7] published annual radiation doses above the lead aprons of 28 radiologists. The average annual dose in their study was $48 \mathrm{mSv}$. The average annual dose in the study by Niklason et al. was higher than the average annual dose in the present study: $45 \mathrm{mSv}$. However, the average annual dose found by Niklason et al. was lower than the average annual dose when radiologist 5 was excluded. The average annual dose of the seven remaining radiologists was $50 \mathrm{mSv}$. 
In the studies by Williams et al. and Nicklason et al. the doses under the lead apron were also measured. Williams et al. found that the average monthly dose under the lead apron ranged from 0 to $0.48 \mathrm{mSv}$. As for the results above the lead apron, Williams et al. reported that the highest average dose was caused by the differences in the nature of work of one of the radiologists. The average monthly dose under the lead apron of all other radiologists (the radiologist with the highest dose was excluded) was $0.08 \mathrm{mSv}$. In the present study the doses under the lead apron varied between 0.06 and $0.2 \mathrm{mSv}$ in a 4 -week period. The average dose, $0.12 \mathrm{mSv}$, was higher than the average dose reported by Williams et al. The differences between the two studies are possibly caused by the differences in thicknesses of the lead aprons between them. Williams et al. reported that the doses were measured under lead aprons of 0.35 and $0.5 \mathrm{~mm}$, while in the present study doses were measured under a layer of $0.25 \mathrm{~mm}$.

Niklason et al. measured doses under lead aprons of 0.25 and $0.5 \mathrm{~mm}$. As for the doses measured above the lead apron, the doses under the lead apron were annual doses. The average annual dose under the lead apron reported by Niklason et al. was $0.88 \mathrm{mSv}$, while in the present study the average annual dose was $1.6 \mathrm{mSv}$ (median, $1.3 \mathrm{mSv}$ ). As for the results reported by Williams et al., the differences in doses were probably caused by the variation in thickness of the lead aprons worn by the radiologists.

In the study reported by Williams et al., the radiologists ensured that the personal dosimeters were worn in all cases. However, the personal dosimeters under the lead aprons were worn not only during interventional procedures but also during other radiology procedures. The authors described that the dedication of the radiologists to wearing additional dosimeters under the lead apron was probably less consistent than for the other dosimeters. In the present study the dedication of the radiologists was not tested, as the dosimeters were simultaneously worn in the special holder. Moreover, the dosimeters in this study were worn exclusively during interventional procedures.

The duration of the measurements in the study by Niklason et al. was approximately 2 months, while in the present study the doses of the majority of the radiologists were measured during 3 consecutive years.

The regression analysis revealed a linear relationship between the doses measured above and those measured under the lead apron. The relation was described by the formula $Y=0.036 X-0.004$. The slope of the line $(0.036)$ is a measure of the transmission of the lead aprons. In the present study a transmission of $3.6 \%$ was found for lead aprons of $0.25 \mathrm{~mm}$. This transmission was derived breasthigh, at the height of the lead collar of radiologists. According to Kharrati et al. [8], the energy of the scattered radiation at the height of the lead collar of radiologists is $\sim 70 \mathrm{kVp}$. The transmission fraction in the present study $(3.6 \%)$ is in accordance with the data on transmission factors of different primary beam energies found by other authors. Murphy et al. [9] found a transmission factor of $3 \%$ for lead aprons of $0.3 \mathrm{~mm}$ in the primary beam of $80 \mathrm{kVp}$. Vano et al. [5] found a fraction transmitted through $0.25-\mathrm{mm}$ lead aprons of between $3.3 \%$ at $70 \mathrm{kVp}$ and $5.7 \%$ at $80 \mathrm{kVp}$. Christodoulou et al. [10] reported about transmission fraction through lead aprons of $0.25 \mathrm{~mm}$ at a tube potential of $70 \mathrm{kVp}$. The transmission fraction ranged from $4.3 \%$ up to $10 \%$.

From the formula $Y=0.036 X-0.004$ the intercept of the regression line can be calculated. The intercept with the $X$-axis lies at $0.11 \mathrm{mSv}$. This value theoretically represents the threshold of the doses above the lead apron before doses under the lead apron. This means that for the doses above the lead apron of $\leq 0.11 \mathrm{mSv}$, only doses below the diction limit are expected. The doses will be reported as $<0.01 \mathrm{mSv}$. As doses up to $0.005 \mathrm{mSv}$ are below the detection limit of the dosimeters and are reported as doses $<0.01 \mathrm{mSv}$, the theoretical threshold is even higher: $0.25 \mathrm{mSv}$, versus $0.11 \mathrm{mSv}$ for the doses above the lead apron. As a matter of fact, in the present study for all measurements above the lead apron $<0.25 \mathrm{mSv}$, the corresponding doses under the lead apron were reported to be below the detection limit of $0.01 \mathrm{mSv}$. The lowest dose in this study above the lead apron for which a dose under the lead apron of $0.01 \mathrm{mSv}$ reported was $0.59 \mathrm{mSv}$.

The minimum thickness recommended by the IAEA [11] for lead aprons of radiologists performing interventional procedures is $0.35 \mathrm{~mm}$. In the present study the doses were measured at $0.25 \mathrm{~mm}$. The doses were measured under one layer of wrap-around lead aprons. Due to the overlap of these aprons, the thickness of the lead apron in the front of the radiologists was $0.5 \mathrm{~mm}$. For practical reasons it was decided to measure the doses under one layer of $0.25 \mathrm{~mm}$ despite the fact that the doses measured were higher than the occupational doses to the body of the radiologists. The occupational doses of the radiologists under the lead apron in this study are therefore overestimated.

The present study showed that additional monitoring under the lead apron is not necessary to estimate the occupational exposure more accurately. This result is in agreement with the conclusions of Schultz et al. [12], based on dose calculations with Monte Carlo simulations. A transmission of $3.6 \%$ for lead aprons $\geq 0.25 \mathrm{~mm}$ does not underestimate the exposure for radiologists.

In the present study the doses under the lead apron were determined almost at the same place as the doses above the lead apron. This was possible as a special holder was used. Before the special holder was introduced it was not possible to derive a relation between the doses above and those under the lead apron. 


\section{Conclusion}

The aim of the present study was to determine whether a relation exists between the doses measured above the lead apron and those measured under the lead apron of radiologists performing interventional procedures and whether two personal dosimeters provide additional information compared to a single dosimeter. It might be concluded that a linear relation between the measurements above and those under the lead apron is proved in this study. With this, there is no evidence for a more accurate estimation of the effective dose when an additional dosimeter is used.

As the results of this study were derived from measurements by radiologists performing interventional procedures, it is not known whether a factor of 0.036 is applicable for other specialists performing interventional procedures, such as interventional cardiologists and electrophysiologists. For this reason, more investigation is needed to derive correction factors for other physicians performing interventional procedures.

Open Access This article is distributed under the terms of the Creative Commons Attribution Noncommercial License which permits any noncommercial use, distribution, and reproduction in any medium, provided the original author(s) and source are credited.

\section{References}

1. Hidajat N, Wust P, Felix R, Schröder RJ (2006) Radiation exposure to patient and staff in hepathic chemoembolization: risk estimation of cancer and deterministic effects. CardioVasc Interv Radiol 29:297-796

2. ICRP (2000) Avoidance of radiation injuries from medical interventional procedures. ICRP Publication 85. Pergamon Press, Oxford

3. Dijk JWE, Julius HW (1996) Dose thresholds and quality assessment by statistical analysis of routine individual monitoring TLD data. Radiat Prot Dosim 66:17-22

4. Renaud L (1992) A 5-y follow-up of the radiation exposure to inroom personnel during cardiac catheterization. Health Phys 62(1): $10-15$

5. Vañó E, González L, Guibelalde E, Fernández JM, Alfonso F, Macaya C (2006) Occupational radiation doses in interventional cardiology: a 15-years follow-up. Br J Radiol 79:383-388

6. Williams JR (1997) The interdependence of staff patient doses in interventional radiology. Br J Radiol 70:498-503

7. Niklason L, Marx M, Chan H (1994) The estimation of occupational effective dose in diagnostic radiology with two dosimeters. Health Phys 67(6):611-615

8. Kharrati H, Agrebi A, Karaoui M (2007) Monte Carlo simulation of x-ray buildup factors of lead its applications in shielding of diagnostic X-ray facilities. Med Phys 34(4):1398-1404

9. Murphy PH, Wu Y, Glaze SA (1993) Attenuation properties of lead composite aprons. Radiology 186:269-272

10. Christodoulou EG, Goodsitt MM, Larson SC, Darner KL, Satti JS, Chan H (2003) Evaluation of the transmitted exposure through lead equivalent aprons used in a radiology department, including the contribution from backscatter. Med Phys 30(6): 1033-1038

11. IAEA (2004) Personal protective equipment. IAEA-PRTM-5. IAEA, Vienna

12. Schultz FW, Zoetelief J (2006) Estimation effective dose for a cardiac catheterisation procedure with single or double personal dosimetrie. Radiat Prot Dosim 118(2):196-204 